\title{
Das novellierte Klimaschutzgesetz
}

\section{Walter Frenz}

(c) Der/die Autor(en) 2021. Dieser Artikel ist eine Open-Access-Publikation.

So sensationell wie der BVerfG-Klimabeschluss war, so rasch reagierte der Gesetzgeber mit der KSG-Novelle, die am 24. 6. 2021 im Bundestag und am 25.6.2021 im Bundesrat verabschiedet wurde. Wurde damit dem BVerfG in vollem Umfang entsprochen?

\section{BVerfG-Klimabeschluss und seine Umsetzung in der KSG-Novelle}

\subsection{Begrenzte Beanstandung des KSG}

Der bereits von diverser Seite gewürdigte ${ }^{1}$ BVerfG-Klimabeschluss vom 24.3.2021 führte zu einer alsbaldigen Novelle des KSG, die über die Vorgaben des BVerfG teilweise hinausging, indem auch der $\mathrm{CO}_{2}-\mathrm{Re}-$ duktionszielwert bis 2030 von $55 \%$ auf $65 \%$ angehoben wurde. Vom BVerfG beanstandet wurden die Regelungen der $\$ 3$ Abs. 1 Satz 2 und $\$ 4$ Abs. 1 Satz 3 KSG 2019 i. V.m. Anlage 2 insoweit, als sie keine $\mathrm{CO}_{2}$-Minderungsziele für Zeiträume ab dem Jahr 2031 enthielten. Dies wurde mit der KSG-Novelle durch die neue Anlage 3 zu $\$ 4$ KSG behoben.

Die insoweit bereits Festlegungen durch die Vorgabe von Jahresemissionsmengen nach \$4 Abs. 1 KSG 2019 vorsehende Verordnungsermächtigung des $₫ 4$ Abs. 6 KSG 2019 genügte dem BVerfG weder zeitlich noch inhaltlich; am besten sollte der Gesetzgeber selbst die erforderlichen normativen Regelungen treffen. ${ }^{2}$ Der Gesetzgeber legte nunmehr die Jahresminderungsziele auch von 2031 bis 2040 als Rahmen für die entsprechenden Jahresemissionsmengen fest; vor allem dadurch wurde die Verordnungsermächtigung deutlich geschärft.

Damit reagierte der Gesetzgeber bereits vor der gesetzten Frist bis zum 31.12.2022; so lange mussten daher die bisherigen Bestimmungen des KSG auch nicht mehr anwendbar bleiben, was bis zum Inkrafttreten einer Neuregelung notwendig war, damit überhaupt eine Reduktionsfestlegung existierte. $^{3}$

\subsection{Reduktionspflichten bis 2030}

Die Regelungen im KSG 2019 mit Pflichten bis 2030 waren hingegen nach dem BVerfG verfassungsgemäß. Sie ermöglichten (noch) die Einhaltung des Ziels, die Erwärmung der Erde auf deutlich unter 2 Grad Celsius und möglichst auf 1,5 Grad Celsius zu begrenzen, und entsprachen daher dem verfassungsrechtlichen Klimaschutzgebot nach Art. 20a GG angesichts des dem Gesetzgeber zugebilligten Gestaltungsspielraums. ${ }^{4}$ Danach waren die Jahresemissionsmengen bis zu diesem Zeitpunkt nicht nachzubessern. Der bisherige Fahrplan hätte damit insoweit bleiben können, auch wenn das BVerfG darauf verweist, dass das von ihm auf der Basis des Sachverständigenrates für Umweltfragen zugrunde gelegte deutsche Restbudget von 6,7 Gigatonnen $\mathrm{CO}_{2}-$ Ausstoß ab 2020 praktisch schon bald nach 2030 aufgebraucht sein wird - und zwar noch ohne Berücksichtigung der $\mathrm{CO}_{2}$-Emissionen aus Landnutzung, Landnutzungsänderung und Forstwirtschaft sowie anteiligem Luft- und Seeverkehr. ${ }^{5}$ Der Sektor Landnutzung, Landnutzungsän-

Prof. Dr. jur. Walter Frenz, Maitre en Droit Public, lehrt Berg-, Umwelt- und Europarecht an der RWTH Aachen, Aachen, Deutschland derung und Forstwirtschaft (LULUCF) wurde nunmehr in einem neuen $₫ 3$ a KSG aufgenommen. ${ }^{6}$

Wenn eine solche Ausschöpfung des deutschen $\mathrm{CO}_{2}$ Restbudgets bis 2031 oder auch (etwas) später droht, müssten die dann lebenden Generationen praktisch jeden weiteren $\mathrm{CO}_{2}$-Ausstoß einstellen, um das deutsche Gesamtbudget nicht $\mathrm{zu}$ überschreiten. Sie würden dann gegebenenfalls unzumutbar belastet. Daher erscheint es inkonsistent, dass das BVerfG trotz dieses Befundes einer weitgehenden Erschöpfung des $\mathrm{CO}_{2}$-Gesamtbudgets kurz nach 2030 keine verstärkte Pflichtenwirkung für die Zeit vor 2030 bejaht und nicht auch insoweit Nachschärfungen verlangt. ${ }^{7}$ Das BVerfG schließt vielmehr nicht aus, dass das konkrete nationale Klimaschutzinstrumentarium so fortentwickelt wird, dass das Minderungsziel bis 2030 eingehalten wird, und dieses als solches genügt ihm. ${ }^{8}$

Letztlich ist dies auch die Konsequenz, dass das BVerfG kein verbindliches $\mathrm{CO}_{2}$-Restbudget ableitet, sondern dem Gesetzgeber insoweit Freiraum lässt: „Die Unsicherheit über die zur Wahrung der Temperaturschwelle global und national verbleibenden Emissionsmöglichkeiten ist derzeit $\mathrm{zu}$ groß, als dass die vom Sachverständigenrat ermittelte Budgetgröße ein zahlengenaues Maß für die verfassungsgerichtliche Kontrolle bieten könnte." "Gleichwohl verweist das BVerfG auf die plausible und nicht zu strenge Bestimmung durch den Sachverständigenrat für Umweltfragen (Begrenzung des Erdtemperaturanstiegs auf 1,75 Grad Celsius und Anteil Deutschlands von 1,1\% an der Weltbevölkerung) ${ }^{10}$ und legt sie den weiteren Überlegungen zugrunde ${ }^{11}$ sowie verlangt seine Berücksichtigung, ohne dass es wohl bis 2030 überzogen wird. ${ }^{12}$ Nur wegen der bisherigen Unsicherheiten in der Ermittlung und Bewertung erfolgt keine rechtlich verbindliche Verankerung. Diese kann aber später jederzeit kommen, wenn nämlich die Unsicherheiten verschwunden sind, und ist im Klimabeschluss des BVerfG sehr wohl angelegt.

Wenn unmittelbar nach 2030 eine Überschreitung des für Deutschland vom BVerfG seinen Überlegungen faktisch zugrunde gelegten $\mathrm{CO}_{2}-$ Budgets eintritt, wird im

1) Beckmann, UPR 2021, $241 \mathrm{ff}$; Calliess, ZUR 2021, $355 \mathrm{ff}$; Faß bender, NJW 2021, $2085 \mathrm{ff}$; Frenz, DVB1. 2021, $810 \mathrm{ff}$; Muckel, JA 2021, 610ff.; Ruttloff/Freihoff, NVwZ 2021, 917 ff.; Schlacke, NVwZ 2021, 912 ff.

2) BVerfG, Beschl. v. 24.3.2021 - 1 BvR 2656/18 u. a., ECLI:DE:BVerfG:2021:rs20210324.1bvr265618, Rdnr. 258ff.

3) S. BVerfG, Beschl. v. 24.3.2021 - 1 BvR 2656/18 u.a., Rdnr. $267 f$.

4) BVerfG, Beschl. v. 24.3.2021 - 1 BvR 2656/18 u.a., Rdnr. 214, $230 \mathrm{ff}$.

5) BVerfG, Beschl. v. 24.3.2021 - 1 BvR 2656/18 u. a., Rdnr. 233 und auch Rdnr. 246.

6) S.u. 2.6.

7) Anders Faßbender, NJW 2021, 2085, 2090 gegen Frenz, EnWZ 2021, 201, 202

8) BVerfG, Beschl. v. 24.3.2021 - 1 BvR 2656/18 u. a., Rdnr. 238

9) BVerfG, Beschl. v. 24.3.2021 - 1 BvR 2656/18 u.a., Rdnr. 236 a.E.

10) Dazu krit. Frenz, Grundzüge des Klimaschutzrechts, 2. Aufl. 2022 i. E., Rdnr. 30 ff.

11) BVerfG, Beschl. v. 24.3. 2021 - 1 BvR 2656/18 u.a, Rdnr. 229 (,,Rechnung tragen“), 237.

12) BVerfG, Beschl. v. 24.3.2021 - 1 BvR 2656/18 u. a., Rdnr. 225 , $230 \mathrm{ff}$., 235 
Tab. 1

\begin{tabular}{lcccccccccc}
\hline & $\mathbf{2 0 3 1}$ & $\mathbf{2 0 3 2}$ & $\mathbf{2 0 3 3}$ & $\mathbf{2 0 3 4}$ & $\mathbf{2 0 3 5}$ & $\mathbf{2 0 3 6}$ & $\mathbf{2 0 3 7}$ & $\mathbf{2 0 3 8}$ & $\mathbf{2 0 3 9}$ & $\mathbf{2 0 4 0}$ \\
Jährliche Minderungsziele gegenüber 1990 & $67 \%$ & $70 \%$ & $72 \%$ & $74 \%$ & $77 \%$ & $79 \%$ & $81 \%$ & $83 \%$ & $86 \%$ & $88 \%$ \\
\hline
\end{tabular}

Ergebnis gleichfalls nicht genug für den Klimaschutz getan und den jungen Generationen eine allzu große Minderungslast aufgebürdet. Daher war eigentlich in der Begründung des BVerfG angelegt, Pflichten auch bis $2030 \mathrm{zu}$ schärfen, wie dies nun erfolgte: Das $\mathrm{CO}_{2}$-Reduktionsziel bis dahin wurde auf $65 \%$ angehoben $(\$ 3$ Abs. 1 Nr. 1 KSG) und die Jahresemissionsmengen der Sektoren bis zu diesem Zeitpunkt nach $\ 4$ Abs. 1 KSG i. V.m. Anlage 2 wurden weiter abgesenkt - insbesondere für die Energiewirtschaft und Industrie als Sektoren mit den höchsten Emissionen. ${ }^{13}$

\subsection{Reduktionspflichten ab 2030}

\subsubsection{Konkreter Minderungspfad bis 2040}

Jedenfalls betrachtete das BVerfG $₫ 3$ Abs. 1 Satz 2 und $₫ 4$ Abs. 1 Satz 3 KSG 2019 i. V.m. Anlage 2 insoweit als verfassungswidrig, ,,als sie die derzeit nicht hinreichend eingedämmte Gefahr künftiger Grundrechtsbeeinträchtigungen begründen." 14 Es bedarf daher Vorkehrungen zur grundrechtsschonenden Bewältigung der nach 2030 drohenden Reduktionslast, und zwar bereits jetzt. ${ }^{15}$

Welche Vorkehrungen $\mathrm{zu}$ treffen sind, überlässt das BVerfG dem Gesetzgeber mit seinem Gestaltungsspielraum. Dieser wird aber erheblich durch die Maßgaben des BVerfG beschränkt. Dabei muss der Gesetzgeber bei seinen Reduktionsmaßgaben eine besondere Sorgfaltspflicht unter Einbeziehung von Vorsorgemaßnahmen wahren. ${ }^{16}$

Der Gesetzgeber muss wegen der notwendigen vorausschauenden Schonung künftiger Freiheit erforderliche Entwicklungs- und Umsetzungsprozesse frühzeitig einleiten und so auch für die Zeit nach 2030 genügend Orientierung geben, um einen hinreichenden Entwicklungsdruck sowie Planungssicherheit zu erzeugen.

Damit mussten $₫ 3$ Abs. 1 Satz 2 und $₫ 4$ Abs. 1 Satz 3 KSG 2019 i. V.m. Anlage 2 um Pflichten für die Zeit nach 2030 erweitert werden. Das ist im KSG durch die darauf bezogene Novelle partiell bereits erfolgt. Bis 2040 geht es um eine $\mathrm{CO}_{2}$-Reduktion um $88 \%$ ( $\$ 3$ Abs. 1 Nr. 2 KSG). In Anlage $3 \mathrm{zu} \ 4 \mathrm{KSG}$ sind die jährlichen Minderungsziele für die Jahre 2031 bis 2040 aufgeführt. Dadurch entsteht ein konkreter Minderungspfad bis 2040, wie vom BVerfG nahegelegt. ${ }^{17}$ Die entsprechenden näheren Maßnahmen, um diese Zielwerte sicherzustellen, müssen ausweislich $\int 9$ KSG die Ministerien bestimmen und am besten in Initiativen für Sachgesetze wie Verschärfungen des GEG für eine Reduktion im Gebäudesektor in die Wege leiten. Die Jahresemissionsmengen von 2031 bis 2040 sind 2024 durch Verordnung nach dem novellierten $\$ 4$ Abs. 6 KSG festzulegen.

\subsubsection{Angepasste Gesetzestechnik}

Die bisherige Grundlage für Fortschreibungen der Emissionsminderungen in Gestalt der Verordnungsermächtigung in $\ 4$ Abs. 6 KSG 2019, durch welche die jährlichen Minderungszeiträume ab dem Jahr 2031 geregelt wurden $(\mathbb{S} 4$ Abs. 1 Satz 5 KSG 2019), genügte nicht. Das gilt zunächst zeitlich: Es war erst 2025 eine Rechtsverordnung der Bundesregierung für weitere Zeiträume nach dem Jahr 2030 mit jährlich absinkenden Emissionsmengen vorgesehen. Die Anschlussfestlegungen an die Jahresemissionsmengen bis 2030 nach $₫ 4$ Abs. 1 Satz 3 KSG i. V.m. Anlage 2 müssen früher erfolgen; zudem genügt nicht eine einmalige Fixierung, sondern es bedarf einer Festlegung in bestimmten Zeitabständen, die normativ zu fixieren sind. ${ }^{18}$
Am ehesten präferiert das BVerfG ohnehin eine Regelung im KSG selbst ${ }^{19}$ - etwa durch eine Fortschreibung der Jahresemissionsmengen nach $₫ 4$ Abs. 1 Satz 3 KSG i. V.m. Anlage 2. Diese erfolgte zwar nicht, aber es wurde durch jährliche Minderungsziele bis 2040 ein konkreter Minderungspfad geschaffen, der zugleich den „Rahmen“20 für die Festschreibung von Jahresemissionsmengen durch den Verordnungsgeber bildet, die bis $2024 \mathrm{zu}$ erfolgen hat. Dieser Minderungspfad gestaltet sich nach Anlage 3 zum KSG wie in Tabelle 1 zu sehen. ${ }^{21}$

Die Jahresemissionsmengen von 2041 bis 2045 werden dann spätestens bis 2034 festgelegt. Damit wurden die wesentlichen Festlegungen für den Verordnungsgeber im neuen $₫ 4$ Abs. 6 KSG geschaffen. Nach dem BVerfG muss nämlich der Gesetzgeber selbst Regelungen zur Größe der Jahresemissionsmengen treffen, ${ }^{22}$ und zwar wegen Art. 80 Abs. 1 GG und des Grundsatzes des Gesetzesvorbehaltes. ${ }^{23}$

\subsection{Notwendige Weiterungen}

Allerdings wurde bisher nur der Rahmen nach dem KSG geändert. Entscheidend ist die Einleitung des vom BVerfG vorausgesetzten umfassenden Umgestaltungsprozesses sowohl im wirtschaftlichen als auch im privaten Bereich, der bei später Festlegung zu - verfassungswidrigen - Grundrechtseinschränkungen der jüngeren Generationen und gegebenenfalls zu einer alsbaldigen Unterbindung $\mathrm{CO}_{2}$-relevanter Tätigkeiten im Wesentlichen führt. ${ }^{24}$ Dieser Umbau betrifft Produkte, Dienstleistungen, Konsumgewohnheiten etc. ${ }^{25}$ und wird nur gelingen, wenn erhebliche Veränderungsprozesse auch in Sachbereichen eingeleitet werden so in der Kreislaufwirtschaft, welche eine mittlerweile anerkannte hohe Bedeutung für den Klimaschutz hat. ${ }^{26}$ Daher bedarf es auch in anderen Gesetzen anpassender Normierungen, die diesen Umwandlungsprozess einleiten.

\section{Nationale Klimaschutzziele}

\subsection{Systematik und Entwicklung}

Das Ziel der Klimaneutralität ist im KSG mittlerweile fest verankert und auf 2045 vorgezogen. Es bedurfte der Anpassung der Zwischenziele, auch um die EU-Vorgaben einzuhalten. ${ }^{27} \mathrm{Um}$ diese Ziele zu erreichen, wurden sektorenbezogen Jahresemissionsmengen festgelegt. Diese sinken

13) Begründung zum Regierungsentwurf, BT-Drs. 19/30230, S. 23

14) BVerfG, Beschl. v. 24.3.2021 - 1 BvR 2656/18 u. a., Rdnr. 243.

15) BVerfG, Beschl. v. 24.3.2021 - 1 BvR 2656/18 u. a., Rdnr. 245 ff. Dazu bereits Frenz, EnWZ 2021, 201, $202 \mathrm{f}$.

16) BVerfG, Beschl. v. 24.3.2021 - 1 BvR 2656/18 u. a., Rdnr. 229

17) Begründung zum Regierungsentwurf, BT-Drs. 19/30230, S. 12

18) BVerfG, Beschl. v. 24.3. 2021 - 1 BvR 2656/18 u. a., Rdnr. $257 \mathrm{f}$

19) BVerfG, Beschl. v. 24.3. 2021 - 1 BvR 2656/18 u. a., Rdnr. 258 a.E.

20) Begründung zum Regierungsentwurf, BT-Drs. 19/30230, S. 12

21) S. bereits Begründung zum Regierungsentwurf. BT-Drs. 19/30230, S. 11

22) BVerfG, Beschl. v. 24.3. 2021 - 1 BvR 2656/18 u. a., Rdnr. 259

23) BVerfG, Beschl. v. 24.3.2021 - 1 BvR 2656/18 u. a., Rdnr. $260 \mathrm{ff}$

24) BVerfG, Beschl. v. 24.3. 2021 - 1 BvR 2656/18 u. a., Rdnr. 249

25) BVerfG, Beschl. v. 24.3.2021 - 1 BvR 2656/18 u. a., Rdnr. 184, 186, 249.

26) Näher Frenz, Grundzüge des Klimaschutzrechts, 2. Aufl. 2022 i. E., Rdnr. $540 \mathrm{ff}$.

27) Begründung zum Regierungsentwurf, BT-Drs. 19/30230, S. 18. 
Tab. 2 Anlage 2 Zulässige Jahresemissionsmengen für die Jahre 2020 bis $2030 \mathrm{zu}$ \&4 KSG 2019:

\begin{tabular}{lrrrrrrrrrrrr}
\hline Jahresemissionsmenge in Mio. Tonnen $\mathbf{C O}_{2}$-Äquivalent & $\mathbf{2 0 2 0}$ & $\mathbf{2 0 2 1}$ & $\mathbf{2 0 2 2}$ & $\mathbf{2 0 2 3}$ & $\mathbf{2 0 2 4}$ & $\mathbf{2 0 2 5}$ & $\mathbf{2 0 2 6}$ & $\mathbf{2 0 2 7}$ & $\mathbf{2 0 2 8}$ & $\mathbf{2 0 2 9}$ & $\mathbf{2 0 3 0}$ & \\
Energiewirtschaft & 280 & & 257 & & & & & & \\
Industrie & 186 & 182 & 177 & 172 & 168 & 163 & 158 & 154 & 149 & 145 & 140 \\
Gebäude & 118 & 113 & 108 & 103 & 99 & 94 & 89 & 84 & 80 & 75 & 70 \\
Verkehr & 150 & 145 & 139 & 134 & 128 & 123 & 117 & 112 & 106 & 101 & 95 \\
Landwirtschaft & 70 & 68 & 67 & 66 & 65 & 64 & 63 & 61 & 60 & 59 & 58 \\
Abfallwirtschaft und Sonstiges & 9 & 9 & 8 & 8 & 7 & 7 & 7 & 6 & 6 & 5 & 5 \\
\hline
\end{tabular}

Tab. 3 Anlage 2 Zulässige Jahresemissionsmengen für die Jahre 2020 bis $2030 \mathrm{zu}$ \4 KSG 2021:28

\begin{tabular}{lrrrrrrrrrrr}
\hline Jahresemissionsmenge in Mio. Tonnen $\mathbf{C O}_{2-}$ Äquivalent & $\mathbf{2 0 2 0}$ & $\mathbf{2 0 2 1}$ & $\mathbf{2 0 2 2}$ & $\mathbf{2 0 2 3}$ & $\mathbf{2 0 2 4}$ & $\mathbf{2 0 2 5}$ & $\mathbf{2 0 2 6}$ & $\mathbf{2 0 2 7}$ & $\mathbf{2 0 2 8}$ & $\mathbf{2 0 2 9}$ & $\mathbf{2 0 3 0}$ \\
Energiewirtschaft & 280 & & 257 & & & & & & & & \\
Industrie & 186 & 182 & 177 & 172 & 165 & 157 & 149 & 140 & 132 & 125 & 118 \\
Gebäude & 118 & 113 & 108 & 102 & 97 & 92 & 87 & 82 & 77 & 72 & 67 \\
Verkehr & 150 & 145 & 139 & 134 & 128 & 123 & 117 & 112 & 105 & 96 & 85 \\
Landwirtschaft & 70 & 68 & 67 & 66 & 65 & 63 & 62 & 61 & 59 & 57 & 56 \\
Abfallwirtschaft und Sonstiges & 9 & 9 & 8 & 8 & 7 & 7 & 6 & 6 & 5 & 5 & 4 \\
\hline
\end{tabular}

mit dem Ansteigen der Ziele. Hier wirkt sich ganz erheblich aus, dass nunmehr das Reduktionsziel in \$3 KSG von $55 \%$ auf $65 \%$ angehoben wurde. Die bislang in Anlage 2 enthaltenen Jahresemissionsmengen wurden dementsprechend nunmehr deutlich verringert, wie die folgende $\mathrm{Ge}-$ genüberstellung zeigt (vgl. Tabelle 2 und 3).

\section{$2.265 \%$ bis 2030 und $88 \%$ bis 2040 als Mindestwerte}

Die nationalen Klimaschutzziele sind näher in $\$ 3$ KSG geregelt, das übergeordnete Ziel der Klimaneutralität ergibt sich nunmehr klar und ohne Relativierung bereits aus $\$ 1$ KSG. In $\$ 3$ Abs. 2 KSG wird es bis 2045 jahresbezogen festgeschrieben; nach 2050 sind negative Treibhausgasemissionen avisiert. Bis 2030 gilt es, die Treibhausgasemissionen im Vergleich zum Jahr 1990 um mindestens $65 \%$ zu mindern, bis 2040 um mindestens $88 \%$ ( $\$ 3$ Abs. $1 \mathrm{KSG})$.

Insoweit handelt es sich daher nicht mehr wie beim bisherigen \$3 Abs. 1 KSG 2019 um einen vorläufigen Schritt, sondern um einen Gesamtplan. Es geht um eine fortdauernde schrittweise Minderung. Dabei können auch staatenübergreifende Mechanismen eingesetzt werden, sofern dies partiell geschieht ( $\$ 3$ Abs. $3 \mathrm{KSG}$ ).

Klimaschutzziele können erhöht, jedoch nicht abgesenkt werden ( $\$ 3$ Abs. 4 Satz 2 KSG). Das ergibt sich außer bei besonderen Umständen, nämlich einem geringeren Schädigungspotenzial der Erderwärmung nach neueren hinreichend gesicherten Erkenntnissen, nach dem Klimabeschluss des BVerfG bereits aus dem Klimaschutzgebot nach Art. 20a GG. ${ }^{29}$

Diese mögliche Erhöhung hat Bedeutung vor allem im Hinblick darauf, dass europäische oder internationale Klimaschutzziele erfüllt werden. Dies könnte insbesondere im Zuge der europäischen Klimaschutzplanung erforderlich werden, so wenn „Empfehlungen“ der Kommission an Deutschland wegen eines vorgelegten nationalen Energieund Klimaplanes ergehen, welche faktisch $\mathrm{zu}$ erfüllen sind. ${ }^{30}$ Die Bundesregierung leitet dann nach $\$ 3$ Abs. 4 Satz 1 KSG die notwendigen Schritte ein, um die bereits festgelegten Zielwerte nach $\$ 3$ Abs. 1 KSG zu erhöhen.

Diese Zielwerte sind also nicht auf Dauer fest verankert, sondern bilden nur einen auch für die Zukunft unabdingbaren Mindestwert. Daher kann dieses Ziel auch nicht wegen der aktuellen Corona-Krise abgesenkt werden. Im Gegenteil wurde sowohl auf europäischer als auch auf nationaler Ebene die Verbindung von Corona und Klimaschutz betont: Die negativen Folgen der Corona-Krise sollen auch und gerade durch Investitionen in den Klimaschutz behoben werden. ${ }^{31}$ Eine Absenkung ist im Übrigen schon insoweit nicht notwendig, als wegen des Rückgangs wirtschaftlicher Aktivitäten während der Corona-Pandemie auch die Treibhausgasemissionen zurückgegangen sein dürften. Das gilt jedenfalls vorerst.

\subsection{Mögliche Verschärfungen}

Eine Verschärfung der nationalen Klimaschutzziele kann sich vor allem im Zuge des Green Deal und des EU-Klimagesetzes sowie dessen Realisierung durch das EU-Klimapaket „Fit for 55“ vom 14. 7.202132 auf europäischer Ebene ergeben. Zwar liegen die deutschen Zielsetzungen konform mit dem auf Deutschland entfallenden Anteil an der Erreichung des europäischen Klimaschutzziels für 2030, ${ }^{33}$ und zwar auch in seiner erhöhten Form: Danach bedarf es eines deutschen Beitrags in Höhe einer Emissionsreduzierung um $62 \%$ bis $68 \%$. $^{34}$

Die Gesetzesbegründung zum KSG 2019 verwies aber bereits auf die fünfjährigen Überprüfungszeiträume entsprechend Art. 4 Abs. 3 des Übereinkommens von Paris auf europäischer Ebene und die gegebenenfalls damit ver-

28) Bereits Begründung zum Regierungsentwurf, BT-Drs. 19/30230, S. 10

29) BVerfG, Beschl. v. 24.3.2021 - 1 BvR 2656/18 u. a., Rdnr. 212 a.E.

30) Näher Frenz, Grundzüge des Klimaschutzrechts, 2. Aufl. 2022 i. E., Rdnr. $144 \mathrm{ff}$.

31) Näher Frenz, in: ders., Gesamtkommentar Klimaschutzrecht, 2021, Einf. B: Klimaschutz und Corona, Rdnr. $1 \mathrm{ff}$.

32) Mitteilung der Kommission v. 14. 7.2021 an das Europäische Parlament, den Rat, den Europäischen Wirtschafts- und Sozialausschuss und den Ausschuss der Regionen, „Fit für 55“: auf dem Weg zur Klimaneutralität - Umsetzung des EU-Klimaziels für 2030, COM(2021) 550 final.

33) Begründung zum Gesetzentwurf der Fraktionen der CDU/CSU und SPD zu einem Bundesklimaschutzgesetz, BT-Drs. 19/14337, S. 27.

34) Nach der Schätzung des Klimaexpertenrates, Bericht zur Vorjahresschätzung der deutschen Treibhausgasemissionen für das Jahr 2020 vom 15.2.2021, s. Begründung zum Regierungsentwurf, BT-Drs. 19/30230, S. 18. 
bundenen Verschärfungen, welche dann auch eine Anpassung der deutschen gesetzlichen Klimaschutzziele erforderlich machen könnten. ${ }^{35}$

Die europäischen Zielsetzungen können durch das für die Umsetzung vorherrschende Solidaritätsprinzip (Art. 2 Abs. 2 EU-Klimagesetz) die bisherigen Zielmarken übersteigen und einen anspruchsvollen deutschen Beitrag erforderlich machen, ${ }^{36}$ wird doch gerade im Zuge der CoronaKrise nunmehr sehr stark auf die deutsche Finanz- und Wirtschaftskraft verwiesen, um die Folgen aufzufangen. Eine solche Folgewirkung könnte auch sein, dass es in anderen EU-Staaten notwendig ist, die EU-Klimaschutzziele zu lockern, sodass dann umgekehrt Deutschland anspruchsvollere Zielgrößen schultern müsste. Das EU-Klimapaket vom 14.7.2021 sieht eine Lastenteilungsverordnung ${ }^{37}$ vor, durch die den Mitgliedstaaten neue strengere Emissionssenkungsziele für Gebäude, Verkehr, Landwirtschaft, Abfallwirtschaft und kleine Unternehmen je nach ihren unterschiedlichen Ausgangssituationen und Kapazitäten zugewiesen werden. ${ }^{38}$

\subsection{Operationalisierung durch jährliche Minderungsziele}

Die Operationalisierung der nationalen Klimaschutzziele nach $\$ 3$ KSG erfolgt durch jährliche Minderungsziele mittels der Vorgabe von Jahresemissionsmengen für die Sektoren Energiewirtschaft, Industrie, Verkehr, Gebäude, Landwirtschaft sowie Abfallwirtschaft und Sonstiges nach $\$ 4$ Abs. 1 KSG. Die nähere Abgrenzung dieser Sektoren ergibt sich aus Anlage 1 zum KSG. Die Jahresemissionsmengen für den Zeitraum bis 2030 sind bereits in Anlage 2 zum KSG festgelegt und nunmehr verschärft worden. ${ }^{39}$ Zudem wurden Reduktionsziele bis 2040 in Anlage 3 festgelegt, denen noch Jahresemissionsmengen folgen müssen, und zwar im Verordnungswege nach dem nachgeschärften $\ 4$ Abs. $6 \mathrm{KSG}$. Bevor die zulässigen Jahresemissionsmengen neu festgelegt werden, ist nach dem geänderten $\$ 12 \mathrm{Abs} .3$ Satz 1 Nr. 1 KSG nunmehr eine Stellungnahme des Expertenrats für Klimafragen einzuholen.

Die Emissionsmengen sinken bis 2030 jährlich linear, im Sektor Energiewirtschaft geht es gleichfalls um eine möglichst stetige Absenkung (\$4 Abs. 1 Satz 4 KSG), wobei allerdings nur für 2020, 2022 und 2030 Jahresemissionsmengen festgelegt werden. ${ }^{40}$ Das gilt auch für die anspruchsvolle Neufassung. Die in Anlage 2 festgelegten Jahresemissionsmengen können nach $\$ 4$ Abs. 5 KSG durch eine im Bundesrat nichtzustimmungspflichtige Rechtsverordnung der Bundesregierung mit Wirkung zum Beginn des jeweils nächsten Kalenderjahres geändert werden, um so (angepasste) Klimaschutzziele nach dem KSG sowie unionsrechtliche Anforderungen zu erfüllen. Der Klimaexpertenrat gibt vorher eine Stellungnahme ab. Der Bundestag muss allerdings - anders als bei der sofortigen Änderung nach $₫ 8$ Abs. 2 und $3 \mathrm{KSG}$ - zustimmen. Dies wird fingiert, wenn er sich nicht nach Ablauf von drei Sitzungswochen seit Eingang der Rechtsverordnung mit ihr befasst hat.

Durch eine solche Rechtsverordnung können nicht nur die bis 2030 gesetzlich festgelegten Jahresemissionsmengen flexibilisiert werden, sondern auch künftige Jahresemissionsmengen für die Sektoren fortgeschrieben werden. ${ }^{41}$ Die eigentliche Verordnungsermächtigung für die Festlegung von Emissionsmengen für weitere Zeiträume nach dem Jahr 2030 bildet aber $\$ 4$ Abs. 6 KSG, der nunmehr im Gefolge des BVerfG-Klimabeschlusses sowohl zeitlich als auch inhaltlich nachgebessert wurde.

Eine solche Verordnung hat im Jahr 2024 zu ergehen. Auch sie muss im Einklang mit der Erreichung der Klimaschutzziele des KSG und mit den unionsrechtlichen Anforderungen stehen, ebenso mit den den Rahmen bildenden jährlichen Minderungszielen; dieser konkret festgelegte Reduktionspfad führt inhaltlich nunmehr dazu, dass eine hinreichend konkrete gesetzliche Ermächtigungsgrund- lage vorliegt. ${ }^{42}$ Dabei geht es ebenfalls um eine fortlaufende jährliche Absenkung. Wegen der Bedeutung besteht ein Zustimmungsvorbehalt des Bundestages, ${ }^{43}$ der aber als gewahrt gilt, wenn sich der Bundestag nicht nach Ablauf von sechs Sitzungswochen seit Eingang der Rechtsverordnung mit ihr befasst.

$\mathrm{Ob}$ ab 2031 auf die Zuweisung von zulässigen Jahresemissionsmengen für die einzelnen Sektoren verzichtet werden kann, und die Bundesregierung daher einen entsprechenden Gesetzgebungsvorschlag vorlegt, entscheidet sich nach dem neuen $\$ 4$ Abs. 7 KSG anhand eines Berichts zum Stand und zur weiteren Entwicklung der $\mathrm{CO}_{2}$-Bepreisung innerhalb der EU sowie zu technischen Entwicklungen, den die Bundesregierung 2027 dem Bundestag vorlegt.

\subsection{Keine Einklagbarkeit}

Die so festgelegten Jahresemissionsmengen sind gem. \4 Abs. 1 Satz 9 KSG verbindlich, ebenso nunmehr die jährlichen Minderungsziele, soweit das KSG auf sie Bezug nimmt. Indes werden auf ihre Einhaltung keine subjektiven Rechte oder klagbaren Positionen begründet ( $\$ 4$ Abs. 1 Satz 7 KSG).

Die Vorschrift ist laut Gesetzesbegründung deklaratorisch, ${ }^{44}$ sodass sich nunmehr durch den BVerfG-Klimabeschluss eine subjektive Einforderbarkeit ergeben könnte. Diese besteht danach aber nur auf die rechtzeitige Einleitung und Festlegung von Klimaschutzmaßnahmen, damit nicht junge Generationen in späteren Jahren übermäßig belastet werden: ${ }^{45}$ Wie der Gesetzgeber dies sicherstellt, obliegt seiner Gestaltung, die sich in den vom BVerfG aufgestellten Eckpunkten bewegen muss.

$\mathrm{Zu}$ diesen Eckpunkten gehören aber nicht konkrete Jahresemissionsmengen. Diese festzulegen ist Aufgabe des Gesetz- bzw. des Verordnungsgebers, der dann aber hinreichend konkrete Vorgaben benötigt, ${ }^{46}$ denen $\$ 4$ Abs. 6 KSG mittlerweile genügt. ${ }^{47}$

Auch aus den Zahlen des IPCC und des Sachverständigenrates für Umweltfragen leitet das BVerfG keine konkreten Mengen ab; nur das vom Sachverständigenrat für

35) Begründung zum Gesetzentwurf der Fraktionen der CDU/CSU und SPD zu einem Bundesklimaschutzgesetz, BT-Drs. 19/14337, S. 28

36) S. Frenz, Grundzüge des Klimaschutzrechts, 2. Aufl. 2022 i. E., Rdnr. 110.

37) European Commission, Proposal for a regulation of th he European Parliament and of tt he Council amending Regulation (EU) 2018/842 on binding annual greenhouse gas emission reductions by Member States from 2021 to 2030 contributing to climate action to meet commitments under the Paris Agreement, COM(2021) 555 final.

38) Mitteilung der Kommission v. 14. 7.2021 an das Europäische Parlament, den Rat, den Europäischen Wirtschafts- und Sozialausschuss und den Ausschuss der Regionen, „Fit für 55“: auf dem Weg zur Klimaneutralität - Umsetzung des EU-Klimaziels für 2030, COM(2021) 550 final, S. 7.

39) S. vorstehend 2.1.

40) Begründung zum Gesetzentwurf der Fraktionen der CDU/CSU und SPD zu einem Bundesklimaschutzgesetz, BT-Drs. 19/14337, S. 28.

41) Begründung zum Gesetzentwurf der Fraktionen der CDU/CSU und SPD zu einem Bundesklimaschutzgesetz, BT-Drs. 19/14337, S. 28.

42) Begründung zum Regierungsentwurf, BT-Drs. 19/30230, S. 21.

43) Begründung zum Gesetzentwurf der Fraktionen der CDU/CSU und SPD zu einem Bundesklimaschutzgesetz, BT-Drs. 19/14337, S. 30 .

44) Begründung zum Gesetzesentwurf der Fraktion CDU/CSU und SPD zu einem Bundesklimaschutzgesetz, BT-Drs. 19/14337, S. 28.

45) BVerfG, Beschl. v. 24.3. 2021 - 1 BvR 2656/18 u. a., Ls. 4.

46) BVerfG, Beschl. v. 24.3.2021 - 1 BvR 2656/18 u.a., Rdnr. $259 \mathrm{ff}$

47) S. o. 1.3.2. 
Umweltfragen errechnete $\mathrm{CO}_{2}$-Gesamtbudget von 6,7 Gigatonnen, das auf Deutschland ab 2020 entfallen soll, legt es seinen Überlegungen zugrunde und verlangt seine Berücksichtigung, ohne aber einen hinreichend konkreten Maßstab für die verfassungsrechtliche Kontrolle zu bilden. ${ }^{48}$ Erst recht gilt dies dann für gegebenenfalls daraus abzuleitende Jahresemissionsmengen.

Jahresemissionsmengen sind nur als solche festzulegen, wenn auch so differenziert, dass eine hinreichende konkrete Orientierung entsteht, um den für die Umgestaltung von Produkten und Verhaltensweisen erforderlichen $\mathrm{Pla}-$ nungsdruck zu erzeugen. ${ }^{49}$ Indes folgen daraus keine konkreten Jahresemissionsmengen. Diese müssen nur immer weiter abgesenkt werden, wie schon im KSG 2019 in \$3 Abs. 1 Satz 1, $\$ 4$ Abs. 6 Satz 1 vorgesehen, sowie einen Reduktionspfad weisen, der unter Wahrung des verbleibenden Emissionsbudgets zur Klimaneutralität führt. ${ }^{50}$

Daraus erwächst aber kein Anspruch auf konkrete Werte. Der freiheitsrechtliche Ansatz mit den Grundrechten als Abwehrrechten auch gegen zu hohe Belastungen der jungen Generationen in der Zukunft des BVerfG führt nur dazu, dass zu hohe Jahresemissionsmengen als verfassungswidrig beanstandet werden können - so wie auch Teile des KSG -, nicht aber zur positiven Einforderbarkeit höherer Werte oder auch nur auf Einhaltung der festgelegten Jahresemissionsmengen.

Jahresemissionsmengen sind auch nach dem Klimabeschluss des BVerfG nicht einklagbar. Insoweit hat sich die Rechtsprechung des BVerfG nicht geändert, auch wenn sie einen subjektiven Anspruch auf stärkere Klimaschutzmaßnahmen bejaht - aber nur als solche und nicht in rechtlich verbindlichen konkreten Zahlen und Mengen, die vielmehr der Normgeber festzulegen hat, wenn auch rechtzeitig und hinreichend stark.

Allerdings werden mit den festgelegten Jahresemissionsmengen auch die EU-Klimaziele realisiert. Soweit die auf Deutschland entfallenden Leistungen durch die von der EU-Kommission begleiteten Klima- und Energiepläne ${ }^{51}$ konkretisiert werden und die festgelegten Jahresemissionsmengen deren Ausdruck sind, spiegeln sie die zur Realisierung von EU-Zielen notwendigen nationalen Anstrengungen wider.

Daher hielt der EuGH im Urteil Janecek Luftreinhaltepläne als notwendiges Planungsinstrument für eine effiziente Luftreinhaltung nach den EU-Vorgaben der EU Luftqualitätsrichtlinie für einklagbar. ${ }^{52}$ Dies muss entsprechend für die Einhaltung von Jahresemissionsmengen sowie für ihre Festlegung zur wirksamen Erreichung der auf Deutschland entfallenden, von der Kommission konkretisierten Beitrags im Hinblick auf den EU-Klimaschutz gelten. ${ }^{53}$ Bislang fehlt es daran. Im Gegenteil wurden in Deutschland das $\mathrm{CO}_{2}$-Reduktionsziel und in dessen $\mathrm{Ge}$ folge die Jahresemissionsmengen erst an die ambitionierten EU-Ziele im EU-Klimagesetz angepasst. ${ }^{54}$ \$ 4 Abs. 1 Satz 5 KSG sieht eigens eine Anpassung vor. Bisher besteht daher keine subjektive Einforderbarkeit.

\subsection{Landnutzung: der neue $\int 3 a K S G$}

Die Novelle des KSG trifft nun eine ausdrückliche nähere Regelung für den Beitrag des Sektors Landnutzung, Landnutzungsänderung und Forstwirtschaft zum Klimaschutz. In einem neuen $\$ 3$ a. Damit wird der Bedeutung Rechnung getragen, dass die meisten natürlichen Ökosysteme Kohlendioxid aus der Atmosphäre binden können, indem Kohlenstoff in ihnen gespeichert wird. So können sie zur Begrenzung des Klimawandels beitragen, wenn die Ökosysteme sowie ihre Funktion als Kohlenstoffspeicher und -senke geschützt und gestärkt werden. ${ }^{55}$ Dabei ist eine kontinuierliche Bindung von Kohlendioxid aus der Atmosphäre unabdingbar, wenn die Treibhausgasneutralität erreicht werden soll. Schließlich können die Treibhaus- gasemissionen mit den gegenwärtigen Minderungsoptionen nicht in allen Sektoren und dabei insbesondere nicht in der Landwirtschaft auf Null reduziert werden. ${ }^{56}$ Für die Nettotreibhausgasneutralität bis 2045 sollte die menschlich veranlasste Freisetzung von Treibhausgasen um mindestens $97 \%$ gegenüber dem Basisjahr 1990 gemindert werden, sodass Restemissionen in Höhe von bis zu 37,5 Mio. Tonnen $\mathrm{CO}_{2}$ - Äquivalanten jährlich bleiben, die dann durch die bis dahin mindestens zu erreichenden negative Emissionsbilanz des Sektors Landnutzung, Landnutzungsänderung und Forstwirtschaft kompensiert werden sollte. ${ }^{57}$

Dementsprechend sieht $₫ 3 \mathrm{a}$ Abs. $1 \mathrm{KSG}$ als Mittelwert der jährlichen Emissionsbilanzen des jeweiligen Zieljahres und der drei vorhergehenden Kalenderjahre des Sektors Landnutzung, Landnutzungsänderung und Forstwirtschaft (LULUCF) folgende Verbesserungen vor:

- Minus 25 Mio. Tonnen Kohlendioxid-Äquivalent bis zum Jahr 2030,

- minus 35 Mio. Tonnen Kohlendioxid-Äquivalenten bis zum Jahr 2040,

- und mindestens minus 40. Mio. Tonnen Kohlendioxid-Äquivalenten bis zum Jahr 2045.

Grundlage für diese Emissionsbilanzen sind wiederum die Daten nach $\$ 5$ Abs. 2 Nr. 3 KSG und damit die vom Umweltbundesamt neben den Emissionsdaten vorgenommenen Darstellungen auch für Quellen und Senken von Treibhausgasen. ${ }^{58}$

Verantwortlich für die Einhaltung dieser Ziele ist das aufgrund seines Geschäftsbereichs überwiegend zuständige Bundesministerium und damit das Bundeslandwirtschaftsministerium, welches für die Einhaltung dieser Ziele erforderlichen nationalen Maßnahmen vorzulegen und umzusetzen hat ( 3 a Abs. $2 \mathrm{KSG}$ ), wie dies auch in anderen Bereichen erfolgt; $\$ 4$ Abs. 4 Sätze 3 und 4 KSG gelten entsprechend. Die Bundesregierung kann durch Rechtsverordnung, welche nicht im Bundesrat zustimmungspflichtig ist, diesen Grundsatz näher ausgestalten. Sie kann Regelungen treffen zur Anrechnung und Verbuchung von Quellen und Senken des LULUCF-Sektors nach unionsrechtlichen Vorgaben (Nr. 1), zum Umgang mit und zur Anrechenbarkeit von natürlichen Störungen (Nr. 2), und zwar nach international verwendeten Standards für die Klimaberichterstattung, ${ }^{59}$ und $\mathrm{zu}$ den Methoden und Grundlagen für die Berichterstattung über Treibhausgasemissionen und den Abbau von Kohlendioxid im LULUCF-Sektor. Das betrifft vor allem Regelungen zur Erstellung der jährlichen Emissionsbilanzen nach $\$ 3$ a Abs. $1 \mathrm{KSG}$, wobei den Rahmen die verbindlichen Vorgaben für die Berichterstattung auf EU-Ebene und auf internationaler Ebene bilden. Dementsprechend ist auch eine darauf bezogene Verordnung anzupassen, wenn sich die Vorgaben für die Berichterstattung ändern. ${ }^{60}$ Schließlich können in eine Verordnung mehrere Bestimmungen aufgenommen werden, um Daten der Fernerkundung vor allem mittels Satellitenge-

48) BVerfG, Beschl. v. 24.3.2021 - 1 BvR 2656/18 u.a, Rdnr. 237f

49) BVerfG, Beschl. v. 24.3.2021 - 1 BvR 2656/18 u.a, Rdnr. 254.

50) BVerfG, Beschl. v. 24.3.2021 - 1 BvR 2656/18 u. a., Rdnr. 255.

51) S. Frenz, Grundzüge des Klimaschutzrechts, 2. Aufl. 2022 i. E., Rdnr. $143 \mathrm{ff} ., 214 \mathrm{ff}$.

52) EuGH, Urt. v. 25.7.2008 - C-237/07, Rdnr. 39 - Janecek.

53) S. noch zur bisherigen EU-KlimaschutzVO bereits Frenz, in: Frenz., Gesamtkommentar Klimaschutzrecht, 2021, \4 KSG Rdnr. $38 \mathrm{ff}$

54) Begründung zum Regierungsentwurf, BT-Drs. 19/30230, S. 18 55) Begründung zum Regierungsentwurf, BT-Drs. 19/30230, S. 19 56) Begründung zum Regierungsentwurf, BT-Drs. 19/30230, S. 19 57) Begründung zum Regierungsentwurf, BT-Drs. 19/30230, S. 19 58) Näher dazu Begründung zum Regierungsentwurf, BTDrs. 19/30230, S. 19

59) Begründung zum Regierungsentwurf, BT-Drs. 19/30230, S. 20. 60) Begründung zum Regierungsentwurf, BT-Drs. 19/30230, S. 20. 
stützter Systeme für die Berichterstattung im Bereich des LULUCF-Sektors zu erheben, zu nutzen und auszuwerten. Diese Systeme werden bisher noch nicht hinreichend genutzt, weshalb vor allem kurzfristige Schwankungen des Klimaschutzbeitrags des Sektors nur schwer erfasst werden können, sodass eine nähere Regelung hier Abhilfe schaffen kann und soll. ${ }^{61}$

\section{Fazit}

Die KSG-Novelle hat den BVerfG-Klimabeschluss umgesetzt und teilweise übertroffen - so durch das $\mathrm{CO}_{2}-$ Emissionsreduktionsziel von $65 \%$ bis 2030 und die Klimaneutralität bis 2045. Der erste Eckpunkt ist bereits den ambitionierteren Zielen der Union geschuldet, die nunmehr im EU-Klimagesetz verankert sind und durch das EU-Klimapaket vom 14.7.2021 instrumentalisiert werden. Daraus erwachsen weiter verstärkte Klimaschutzlasten Deutschlands, die wohl alsbald eine neue KSG-Novelle erfordern werden. Zudem hat die Hochwasserkatastrophe von Mitte Juli 2021 in erschreckender Deutlichkeit gezeigt, wie wichtig Klimaschutz ist. Dieser muss daher stärker und schneller werden, auch wenn diese Maßnahmen nicht sofort greifen werden, bildet der Klimawandel doch einen langfristig angelegten Prozess. Dieser muss aber möglichst weitgehend eingefangen werden, damit nicht noch stärkere und häufigere ,,Jahrhunderthochwasser“" sowie andere Kli- maphänomene wie Trockenheit, Wirbelstürme oder vermehrter Hautkrebs drohen, die so lange fern sind, wie sie einen nicht selbst betreffen. Nach der Reform des KSG ist also vor der Reform.

Open Access. Dieser Artikel wird unter der Creative Commons Namensnennung 4.0 International Lizenz veröffentlicht, welche die Nutzung, Vervielfältigung, Bearbeitung, Verbreitung und Wiedergabe in jeglichem Medium und Format erlaubt, sofern Sie den/die ursprünglichen Autor(en) und die Quelle ordnungsgemäß nennen, einen Link zur Creative Commons Lizenz beifügen und angeben, ob Änderungen vorgenommen wurden.

Die in diesem Artikel enthaltenen Bilder und sonstiges Drittmaterial unterliegen ebenfalls der genannten Creative Commons Lizenz, sofern sich aus der Abbildungslegende nichts anderes ergibt. Sofern das betreffende Material nicht unter der genannten Creative Commons Lizenz steht und die betreffende Handlung nicht nach gesetzlichen Vorschriften erlaubt ist, ist für die oben aufgeführten Weiterverwendungen des Materials die Einwilligung des jeweiligen Rechteinhabers einzuholen.

Weitere Details zur Lizenz entnehmen Sie bitte der Lizenzinformation auf http://creativecommons.org/licenses/by/4.0/deed.de.

Open Access funding enabled and organized by Projekt DEAL.

61) Begründung zum Regierungsentwurf, BT-Drs. 19/30230, S. 20.

\title{
Drittschutz beim Vollzug von Raumordnungsplänen?
}

\author{
Zur Anfechtbarkeit von Zielabweichungsbescheiden durch Hoheitsträger und Private \\ Boas Kümper
}

(c) Der/die Autor(en) 2021. Dieser Artikel ist eine Open-Access-Publikation.

\begin{abstract}
Um einen Versto $\beta$ gegen raumordnungsrechtliche Vorgaben auszuschließen, kann die Aufstellung von Bauleitplänen, Regionalplänen und Fachplänen sowie die Zulassung raumbedeutsamer Vorhaben eine Abweichung von den Zielen der Raumordnung nach S6 Abs. 2 ROG erfordern. Der Beitrag untersucht, inwieweit die Zulassung einer Zielabweichung von Umweltschutzvereinigungen, Gemeinden und weiteren öffentlichen Stellen sowie privaten Dritten gerichtlich angegriffen werden kann.
\end{abstract}

\section{Die Zielabweichung als Befreiung von der Bindung an Raumordnungsziele}

Die Ziele der Raumordnung sind als die ,verbindlichen“ Festlegungen der Raumordnungspläne (vgl. \3 Abs. 1 Nr. 2 ROG) das zentrale Steuerungsinstrument der Raumordnungsplanung. ${ }^{1}$ Sie entfalten über die gesetzlichen Raumordnungsklauseln, insbesondere die allgemeine Zielbeachtenspflicht nach $₫ 4$ Abs. 1 ROG und besondere fachgesetzliche Bestimmungen wie die in $\mathbb{\$} 1$ Abs. 4 BauGB normierte Anpassungspflicht, strikte Bindungswirkungen gegenüber den Trägern der Fachplanung und der Bauleitplanung, ${ }^{2}$ außerdem für behördliche Zulassungsentscheidungen nach $\int 4$ Abs. 1 S. 1 Nr. 2 und Nr. 3 ROG sowie nach $\int 4$ Abs. 2 ROG in Verbindung mit dem Fachrecht (z. B. $\ 35$ Abs. 3 S. 2 HS 1 oder S. 3 BauGB). ${ }^{3}$ Die Ziele der

Dr. Boas Kümper, Wissenschaftlicher Referent,

Zentralinstitut für Raumplanung, Universität Münster,

Münster, Deutschland
Raumordnung sind somit der Abwägung oder Ermessensausübung vorgelagerte Vorgaben; die auf sie verpflichteten Planungsträger und Zulassungsbehörden unterliegen ei-

1) Vgl. auch etwa Paetow, Ziele der Raumordnung - eine Rechtsprechungskarriere, in: Appel u.a. (Hrsg.), Festschrift für Rainer Wahl, 2011, S. 511 ff.; Hager, Grundfragen des raumordnerischen Zielsystems in Deutschland, BauR 2011, 1093 ff.; Bartram, Die Ziele der Raumordnung. Ein Planungsinstrument zwischen gewachsenem Steuerungsanspruch und verfassungsrechtlichen Anforderungen, 2012, S. 60 ff., mit zahlreichen Beispielen besonders regelungsintensiver Ziele; Snowadsky, Ziele und Grundsätze der Raumordnung. Eine rechtstheoretische Untersuchung zur Abgrenzung und Abgrenzbarkeit, 2016, S. 49 ff., $70 \mathrm{ff}$.

2) Zur strikten Bindung an die Ziele grundlegend BVerwG, Beschl. v. 20.8.1992 - 4 NB 20.91, BVerwGE 90, 329, 332 ff.; siehe ferner etwa BVerwG, Urt. v. 16.3.2006 - 4 A 1075.04, BVerwGE 125, 116 Rdnr. 68; BVerwG, Urt. v. 16.12.2010 - 4 C 8.10, BVerwGE 138, 301 Rdnr. 7; Goppel, in: Spannowsky/Runkel/Goppel, ROG, 2. Aufl. 2018, $\$ 4$ Rdnr. 23 ff.; Durner, in: Kment (Hrsg.), ROG mit Landesplanungsrecht, 2019, \$4 Rdnr. 58 ff.; Runkel, in: Bielenberg/Runkel/Spannowsky, Raumordnungs- und Landesplanungsrecht des Bundes und der Länder, Stand: Februar 2020, M $\$ 4$ ROG 2017 Rdnr. 153 ff.

3) Weiterführend zur Zielbindung nach den jeweiligen Vorschriften Goppel, in: Spannowsky/Runkel/Goppel, ROG, 2. Aufl. 2018, \$4 Rdnr. 35 ff., 58 ff., 75 ff.; Durner, in: Kment (Hrsg.), ROG mit Landesplanungsrecht, 2019, \$4 Rdnr. 11 ff., 95 ff., 122 ff., 139 ff.; Runkel, in: Bielenberg/Runkel/Spannowsky, Raumordnungs- und Landesplanungsrecht des Bundes und der Länder, Stand: Februar 2020, M \$4 ROG 2017 Rdnr. 73 ff., 289 ff. 